$>$ Nous montrons dans cet article que l'expression des récepteurs de l'angiotensine dans le cerveau est modulée lors d'un stress systémique ou psychologique. Cette plasticité a des caractéristiques distinctes selon la nature de l'agent stresseur, et pourrait contribuer à la genèse des réponses physiologiques compensatoires visant à maintenir l'intégrité de l'organisme. <

\section{Récepteurs de l'angiotensine dans le cerveau et adaptation au stress}

Didier Mouginot, Guy Drolet

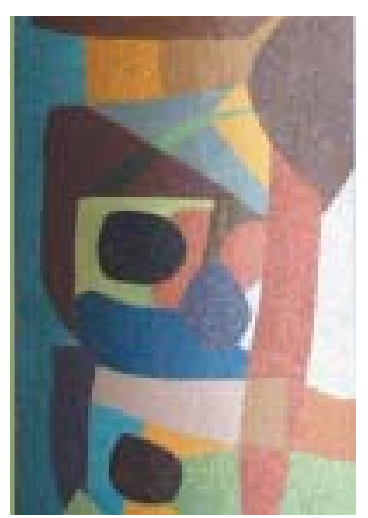

Centre de recherche en neurosciences, CHUL et Université Laval, Faculté de médecine, 2705, boulevard Laurier, Sainte-Foy, Québec, GIV 4G2 Canada. didier.mouginot@crchul.ulaval.ca
Les facteurs de stress peuvent être définis au moins en deux catégories: les facteurs de stress de type social, psychologique ou émotionnel, et les facteurs de stress de type systémique. Dans le premier cas, l'analyse des facteurs de stress requiert la participation des structures limbiques, puisque le cerveau doit d'abord comparer la situation présente avec les expériences antérieures pour déterminer si la situation est stressante ou non et déclencher la réponse physiologique appropriée [1].

La seconde catégorie de facteurs de stress engendre l'activation de l'axe corticotrope indépendamment des structures corticales et du système limbique, et peut mettre en jeu la survie de l'organisme (infection, hémorragie...) [2]. Une interprétation qualitative et émotionnelle de ces facteurs de stress n'est pas nécessaire pour amorcer l'activation de l'axe corticotrope, car l'information est transmise directement vers le noyau paraventriculaire hypothalamique (PVN) via des afférences viscérales excitatrices provenant des structures du tronc cérébral et des organes circumventriculaires.

En choisissant un exemple de stress systémique, la perturbation de l'équilibre hydro-électrolytique, et un exemple de stress psychologique, l'immobilisation, nous émettons l'hypothèse selon laquelle le système rénineangiotensine central, et plus particulièrement la régulation de l'expression des récepteurs centraux de l'angiotensine II, pourrait constituer un mécanisme moléculaire important dans la genèse des réponses physiologiques compensatoires.

\section{La régulation de l'expression des récepteurs $\mathrm{AT}_{1}$ centraux: un processus adaptatif à une perturbation de l'équilibre sodé?}

Dans le contexte de l'homéostasie hydro-électrolytique, des états de déshydratation ou d'hyponatrémie déclenchent des mécanismes osmorégulateurs rapides [3], comme la libération de l'hormone antidiurétique, et des mécanismes régulateurs à plus long terme, comme la synthèse d'angiotensine II (AngII), un peptide agissant au niveau central pour induire la soif et l'appétit pour le sel [4]. L'Angll est produite notamment dans les structures présentant une barrière hémato-encéphalique réduite, telle que l'organe vasculaire de la lame terminale (OVLT) et l'organe subfornical (SFO). L'Angll agit dans le cerveau soit sous sa forme circulante (action endocrine), soit comme neuromodulateur, principalement via les récep- 
teurs de type $1\left(\mathrm{AT}_{1 \mathrm{~A}}\right)$ localisés notamment sur les neurones de la lame terminale et de la division parvocellulaire du PVN (pPVN), qui sont toutes des structures impliquées dans les régulations hydro-électrolytiques et cardiovasculaires.

En utilisant des injections de furosémide, un diurétique de l'anse empêchant la recapture des ions sodium $\left(\mathrm{Na}^{+}\right)$, nous avons montré qu'une hyponatrémie induit

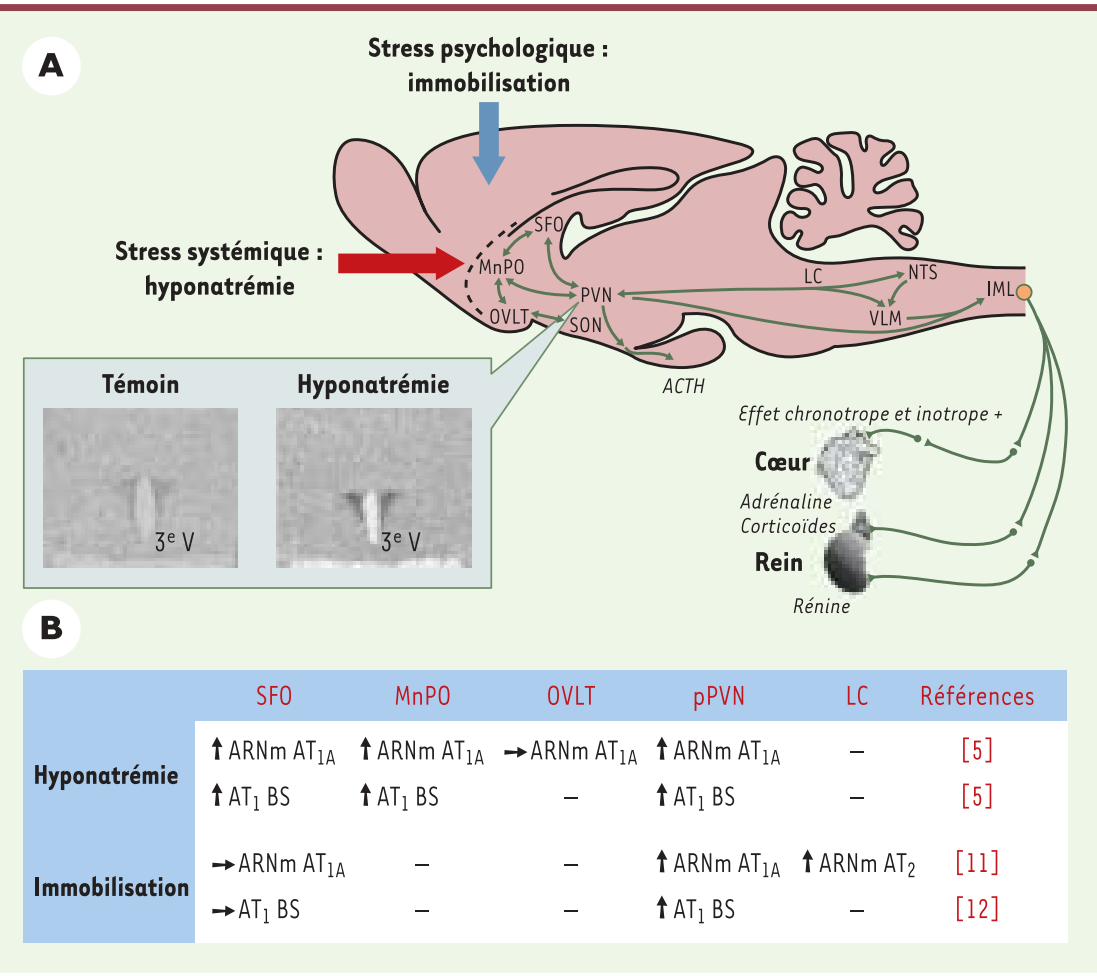

Figure 1. Des facteurs de stress d'origine systémique ou psychologique augmentent l'expression des récepteurs $\boldsymbol{A} T_{1}$ dans différentes structures de l'hypothalamus. A. Schéma représentant les différents noyaux et leurs connexions impliquées dans les réponses à un facteur de stress psychologique (immobilisation) ou systémique (hyponatrémie). Une immobilisation aiguë induit principalement l'activation de l'axe hypothamo-hypophyso-surrénalien. Une hyponatrémie aiguë, surtout détectée au niveau de la lame terminale (SFO, MnPO et OVLT), induit la production d'angiotensine II et d'aldostérone, ce qui favorise l'appétit spécifique pour le sel. Le système rénine-angiotensine central et le noyau paraventriculaire (PVN) semblent impliqués dans les réponses compensatrices, car ces deux types de stress induisent une augmentation de la synthèse des $A R N m$ codant pour le récepteur $A T_{1 A}$ dans la division parvocellulaire du PVN. L'insert illustre cette néosynthèse mesurée 4 heures après l'injection de furosémide $(10 \mathrm{mg} / \mathrm{kg}$, rat Wistar). B. Structures de l'hypothalamus ayant montré une plasticité de l'expression des ARNm $A T_{1 A}$ et de la protéine (récepteur $A T_{1}$ ) après un stress systémique (injections de furosémide) ou psychologique (immobilisation de 90 ou 120 minutes). Noter l'augmentation de la synthèse d'ARNm codant pour le récepteur $\mathrm{AT}_{2}$ dans le locus coeruleus après une immobilisation de 90 minutes. ACTH: corticotrophine; BS: site de liaison; IML: colonne intermédiolatérale; LC: locus coeruleus; $\mathrm{MnPO}$ : noyau préoptique médian; NTS: noyau du tractus solitaire; OVLT: organe vasculaire de la lame terminale; pPVN: division parvocellulaire du noyau paraventriculaire; SFO: organe subfornical; SON : noyau supraoptique; VLM: moelle ventromédiale; $3^{\mathrm{e}} \mathrm{V}$ : $3^{\mathrm{e}}$ ventricule. une augmentation de la synthèse des $A R N m A T_{1 A}$ dans le $\mathrm{SFO}$, le noyau préoptique médian $(\mathrm{MnPO})$ et le $\mathrm{pPVN}$ (Figure 1) [5]. Dans le MnPO, la population de neurones I'ARNm AT ${ }_{1 A}$ demeure stable après le traitement pharmacoque, alors qu'elle est augmentée dans le pPVN, ce qui montre que trémie transitoire se traduit soit par une augmentation de l'expression our l'ARNm $A_{1 A}$, soit par l'activation de ce même gène par des neurones qui l'exprimaient peu ou pas dans des conditions homéostatiques normales. Cette néosynthèse d'ARNm est rapide et se maintient à court terme $(24 \mathrm{~h})$ tant que le déficit n'est pas compensé. Elle s'accompagne d'une augmentation décalée dans le temps du nombre de récepteurs $\mathrm{AT}_{1}$ (sites de liaison) dans ces mêmes noyaux centraux (Figure IB). Un déficit chronique (2 semaines) en $\mathrm{Na}^{+}$est cependant caractérisé par un niveau constant de récepteurs $A T_{1}$ dans les organes circumventriculaires et par leur faible augmentation dans le PVN [6]. Ces études indiquent qu'un déficit transitoire en $\mathrm{Na}^{+}$induit une plasticité de l'expression des récepteurs $A T_{1 A}$ dans les structures du cerveau impliquées dans la régulation de l'équilibre hydro-électrolytique. Cependant, une hyponatrémie chronique impliquerait une régulation de l'expression du gène $A T_{1 A}$ différente ou l'activation de mécanismes post-traductionnels adaptatifs responsables d'une régulation du niveau des récepteurs $\mathrm{AT}_{1}$ dans l'hypothalamus.

Il existerait une relation de cause à effet entre une hypernatrémie soutenue et le développement de maladies cardiovasculaires chez des patients présentant une grande sensibilité au sel. Il a été montré qu'un régime riche en $\mathrm{Na}^{+}$administré pendant plusieurs semaines augmente l'expression des $A R N m A_{1 A}$ dans le cerveau de rats Dahl-S, une souche de rats sélectionnés par croisement qui présente une sensibilité au sel associée au développement d'une hypertension artérielle [7]. Une hypernatrémie soutenue ( 3 semaines) induit une augmentation du nombre de récepteurs $\mathrm{AT}_{1}$ (sites de liaison) dans le pPVN, le MnPO ventral et l'organe vasculaire de la lame termi- 
nale (OVLT) chez les rats Wistar, qui demeurent normotendus après une hypernatrémie chronique. En revanche, les rats Dahl-S devenus hypertendus après un régime enrichi en sel ne présentent pas de régulation des récepteurs $A T_{1}$ dans ces mêmes régions [8]. II semblerait que, contrairement à ce qui a été observé lors d'une hyponatrémie chronique, une hypernatrémie de plusieurs semaines entraînerait une régulation persistante de l'expression des récepteurs $A T_{1}$ dans I'hypothalamus de rats normotendus. Chez les rats Dahl-S, devenus hypertendus, il est intéressant de remarquer que l'activation du gène codant pour les récepteurs $A T_{1 A}$ centraux est déclenchée par l'hypernatrémie, mais que celle-ci ne se traduit pas par une augmentation de l'expression des récepteurs $\mathrm{AT}_{1}$ dans les régions associées au maintien de l'homéostasie hydro-électrolytique et cardiovasculaire.

\section{La réponse au stress chronique induit-elle une plasticité des récepteurs centraux de l'angiotensine?}

L'exposition répétée et prolongée à des facteurs de stress induit l'apparition de mécanismes compensateurs visant à atténuer les effets potentiellement néfastes du stress chronique. Plusieurs données factuelles suggèrent que l'Angll joue un rôle essentiel dans l'élaboration de ces réponses au stress en participant à la régulation des axes corticotrope et autonomique. L'Angll est libérée dans le PVN en réponse à des stimulus physiologiques, suggérant qu'elle agit comme un neurotransmetteur au niveau des axes effecteurs du stress [9]. Elle favoriserait ainsi la libération de l'hormone corticotrope $(\mathrm{ACTH})$, via une activation des neurones à corticolibérine (CRH) du PVN [10], mais elle favoriserait également la libération de rénine en activant l'innervation sympathique du rein. Dans la compréhension des mécanismes cellulaires impliqués dans les réponses au stress, nous nous sommes intéressés à l'expression de l'ARNm codant pour les récepteurs $A T_{1 A}$ en réponse à des expositions aiguës et chroniques à un stress psychologique chez des rats Wistar [11]. Les résultats ont montré qu'un stress d'immobilisation aigu induisait une augmentation rapide de l'expression des $A R N m A_{1 A}$ dans le pPVN (Figure 1B). Cette réponse s'atténue rapidement après la disparition du stress, et les niveaux d'ARNm AT 1 sont comparables au niveau constitutif de ces ARN environ 5 heures après l'induction du stress psychologique. Une immobilisation répétée plusieurs jours (stress chronique) avant l'exposition à un stress aigu homotypique (immobilisation) ou hétérotypique (jet d'air) induit une augmentation de l'expression des
$A R N m A T_{1 A}$ similaire à celle obtenue avec un stress aigu, mais la néosynthèse des $A R N m$ intervient plus rapidement, suggérant qu'un stress chronique ne désensibilise pas les mécanismes de régulation des ARNm mais, au contraire, facilite l'activation du gène codant pour les récepteurs $A T_{1 A}$. Des expériences de radioliaison ont démontré par ailleurs que la néosynthèse des $A R N m A T_{1 A}$ induite lors d'un stress chronique est suivie par une augmentation du nombre de récepteurs $\mathrm{AT}_{1}$ (sites de liaison) dans le PVN [12]. Ainsi, l'exposition répétée à un stress psychologique induit une plasticité de l'expression des récepteurs $A T_{1}$ dans le PVN.

\section{Conclusions}

Nous avons rapporté des éléments montrant que des facteurs de stress d'origine systémique ou psychologique peuvent influencer l'activité du système rénineangiotensine central en réglant de façon précise l'expression des gènes codant pour les récepteurs $A T_{1 A}$. Cette plasticité des récepteurs centraux de l'angiotensine pourrait constituer un mécanisme moléculaire privilégié dans l'élaboration des réponses physiologiques compensatoires au stress. De façon générale, l'activation du gène $A T_{1 A}$ est transitoire pour les facteurs de stress aigu, permettant une réponse adéquate à des stimulus rapprochés. Pour les facteurs de stress chronique, la notion de décours temporel semble être importante. Ce décours serait très rapide lors de stress psychologiques, ce qui permettrait une sensibilisation du système régulateur ou, pour le moins, un mécanisme adaptatif permettant une réponse optimale lors de la convergence de facteurs de stress. Dans le cas d'un stress systémique d'origine hydro-électrolytique, le décours temporel serait lent (hyponatrémie) ou absent (hypernatrémie), suggérant la présence de mécanismes compensateurs soutenus. L'augmentation de l'expression des récepteurs $A T_{1 A}$ serait liée à un effet protecteur face au stress. Ainsi, chez les rats présentant une sensibilité au sel, une absence de régulation de l'expression des récepteurs $A T_{1}$ de l'hypothalamus pourrait être associée au développement de l'hypertension artérielle. $\diamond$
TIRÉS À PART

D. Mouginot 


\section{SUMMARY}

Plastic expression of the brain angiotensin receptors: a molecular mechanism for stress adaptation?

In this short review, we hypothesize that the central renin-angiotensin system might participate to the initiation of compensatory responses to a stressor agent. Regulation of the expression of the brain angiotensin receptors might constitute a primary molecular mechanism by which this protecting action would take place. We illustrate this possibility by investigating the expression of the angiotensin type 1 receptor in the hypothalamus in response to systemic and neurogenic stressors. $\diamond$

\section{RÉFÉRENCES}

1. Herman JP, Cullinan WE. Neurocircuitry of stress: central control of the hypothalamo-pituitaryadrenocortical axis. Trends Neurosci 1997; 20: 78-84.

2. Sawchenko PE, Li HY, Ericsson A. Circuits and mechanisms governing hypothalamic responses to stress: a tale of two paradigms. Prog Brain Res 2000; 122: 61-78. osmoreception and Neuroendocrino 15: $231-74$.

4. Fitzsimons JT. Angiotensin thirst, and sodium appetite. Physiol Rev 1998 ; $78: 583-6$.

5. Charron G, Laforest S, Gagnon C, Drolet G, Mouginot D. Acute sodium deficit triggers plasticity of the brain angiotensin type 1 receptors. FASEB J 2002 ; $16: 610-2$.
6. Yamada H, Mendelsohn FA. Angiotensin II receptor binding in the rat hypothalamus and circumventricular organs during dietary sodium deprivation.

Neuroendocrinology 1989 ; 50: 469-75.

7. Strehlow K, Nickenig G, Roeling J et al. AT(1) receptor regulation in saltsensitive hypertension. Am J Physiol 1999; 277: H1701-7.

8. Mouginot D. Sel et expression des récepteurs centraux de l'angiotensine de type 1. In: $44^{\mathrm{e}}$ Réunion annuelle du Club de recherche clinique du Québec, 2002,

St-Sauveur, Québec, Canada. Med Sci 2002; 18 (suppl): 9.

9. Ferguson AV, Washburn DL, Latchford KJ. Hormonal and neurotransmitter roles for angiotensin in the regulation of central autonomic function. Exp Biol Med 2001; 226: 85-96.
10. Aguilera G, Young WS, Kiss A, Bathia A. Direct regulation of hypothalamic corticotropin-releasinghormone neurons by angiotensin II. Neuroendocrinology 1995; 61: 437-44.

11. Dumont $\varepsilon C$, Rafrafi $S$, Laforest S, Drolet G. Involvement of central angiotensin receptors in stress adaptation. Neuroscience 1999; 93: 877-84.

12. Leong DS, Terron JA, Falcon-Neri A, et al. Restraint stress modulates brain, pituitary and adrenal expression of angiotensin II AT(1A), $\mathrm{AT}(\mathrm{B} B$ ) and $\mathrm{AT}(2)$ receptors. Neuroendocrinology 2002; 75: 227-40. 